\title{
Industrial Robot Trajectory Accuracy Evaluation Maps for Hybrid Manufacturing Process Based on Joint Angle Error Analysis
}

\author{
Zhiyuan Wang*, Renwei Liu, Todd Sparks, Xueyang Chen and Frank Liou \\ Department of Mechanical and Aerospace Engineering, Missouri University of Science and Technology, USA
}

\begin{abstract}
Industrial robots have been widely used in various fields. The joint angle error is the main factor that affects the accuracy performance of the robot. It is important to notice that these kinematic parameters error cannot be eliminated from the robot system completely. Even after calibration, these errors still exist and will be fluctuated during the robot system running. This paper proposed a new method of finding the best position and orientation to perform a specific working path based on the current accuracy capacity of the robot system. By analyzing the robot forward/inverse kinematic and the angle error sensitivity of different joint in the serial manipulator system, a new evaluation formulation is established for mapping the trajectory accuracy within the robot's working volume. The influence of different position and orientation on the movement accuracy of the end effector has been verified by experiments and discussed thoroughly. Finally, a visualized evaluation map can be obtained to describe the accuracy difference of a robotic laser deposition working path at different positions and orientations. This method is helpful for making the maximum usage of the robot's current accuracy ability rather than blindly pursuing the higher accuracy of the robot system.
\end{abstract}

Keywords: Industrial robot; Trajectory accuracy; Joint angle error; Hybrid manufacturing

\section{Introduction}

Usually, the serial robots are mainly used in industry for tasks that require good repeatability $[1,2]$. In this case, the movement accuracy of a robot is not important, as long as the robot end-effector poses are manually taught, repeatability is all that matters. However, in offline programming tasks, like the robotic hybrid manufacturing process, movement accuracy becomes important, since the working path and positions are defined in a virtual space with respect to an absolute or relative coordinate system.

In order to improve the precision of the industrial robot, some researchers focused on the identification of the geometric parameter errors, but ignored the non-geometric errors of the industrial robot system $[3,4]$. These studies assumed that the effect of the non-geometric errors on the robot position errors is ignorable [5-7]. Thus, the identified kinematic parameters based on this method are not accurate, because these non-geometric errors can affect the position accuracy of the end effector greatly.

Other researchers developed the robot kinematic model including geometric and joint compliance errors. Ahmed Joubair and Bonev [8] described a kinematic calibration method using distance and sphere constraints to improve the accuracy of a six-axis serial industrial robot in a specific target workspace. Hudgens et al. [9] proposed a method for the identification of general robot compliance characteristics under applied torques and forces. Judd and Knasinski [10] investigated many error sources of a physical robot, including arms geometric errors, the error of servo, deformation of structure, and the error of installation. Dulen and Schroer [11] adopted the elastic beam theory to study the robot link effects as represented by the changing of six differential elements.

Besides, some researchers used the mapping method to intuitively illustrate the performance of robot's manufacturing capacity. Wang et al. [12] proposed method for mapping the stiffness difference of a robotic deposition working path at different positions and orientations. Ruggiu [13] mapped the stiffness of a translational parallel mechanism using a general formulation based on the development of the principle of virtual work. Pinto et al. [14] used finite element method (FEM), and experimental measurements for the stiffness mapping of a Daedalus I, and concluded that volume FEM was more precise but leads to long calculation times.

It is important to notice that these kinematic parameters error cannot be eliminated from the robot system completely. Even after calibration, these errors still exist and will be fluctuated during the robot system running. Thus it is more meaningful to make the maximum usage of the robot's current accuracy ability rather than blindly pursuit the higher accuracy of the robot system. This paper proposed a new method of finding the best position and orientation to perform a specific working path based on the current accuracy capacity of the robot system. This paper is composed as the following structure: Firstly, by analyzing the angle error sensitivity of different joint in the serial manipulator system, a new evaluation formulation is established for mapping the trajectory accuracy within the robot's working volume. Then the influence of different position and orientation on the movement accuracy of the end effector has been verified by experiments and discussed thoroughly. Finally, a visualized evaluation map can be obtained to describe the accuracy difference of a robotic laser deposition working path at different positions and orientations.

\section{D-H Representation of 6-DOF Industrial Robot}

The Nachi Robot (SC300F-02) is used as an illustrate example

*Corresponding author: Zhiyuan Wang, Department of Mechanical and Aerospace Engineering, Missouri University of Science and Technology, Rolla, Missouri 65409, USA, Tel: 5736128669; E-mail: zwc4b@mst.edu

Received January 09, 2018; Accepted January 24, 2018; Published January 28, 2018

Citation: Wang Z, Liu R, Sparks T, Chen X, Liou F (2018) Industrial Robot Trajectory Accuracy Evaluation Maps for Hybrid Manufacturing Process Based on Joint Angle Error Analysis. Adv Robot Autom 7: 183. doi: 10.4172/2168-9695.1000183

Copyright: $\odot 2018$ Wang Z, et al. This is an open-access article distributed under the terms of the Creative Commons Attribution License, which permits unrestricted use, distribution, and reproduction in any medium, provided the original author and source are credited. 
throughout this paper. It has a $4.1 \mathrm{~m}^{2}$ (cross-section area) operating area and $300^{\circ}$ rotation range for the base motor (Figure 1), which could provide a much bigger working envelope than any current hybrid manufacturing system. The 6-axis movement mechanism makes the deposition/machining process more flexible in building a model with complex features. The kinematic chain of Nachi Robot (SC300F-02) is shown in Figure 2.

When set all the joint angles to be $0^{\circ}$, thus the robot's posture will look like as in Figure 3. Thus, the reference frames representation of Nachi robot as shown in Figure 4.

Hayat et al. [15] discussed the method of identification of D-H parameters of an industrial robot, which can be used to parameterize the Nachi robot. According to the preceding assigned coordinate frames, the parameters of $\mathrm{D}-\mathrm{H}$ model can be filled out in Table 1 .

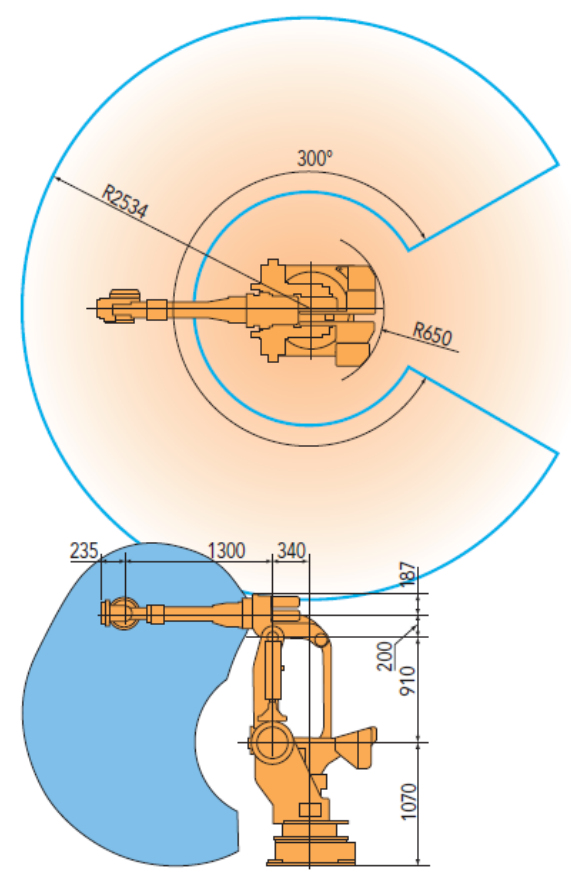

Figure 1: Working envelope and links schematic of Nachi Robot (SC300F-02).

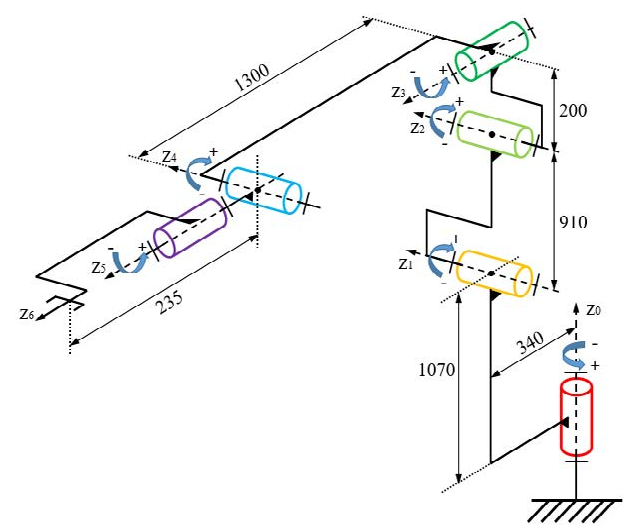

Figure 2: Kinematic chain schematic of Nachi Robot (SC300F-02)

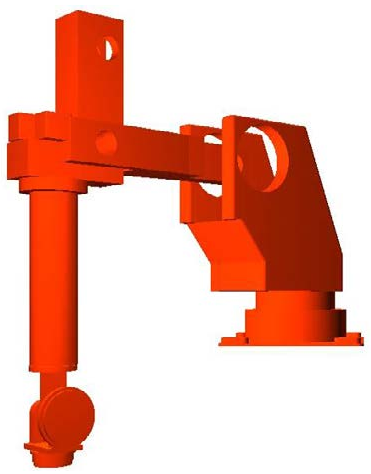

Figure 3: Robot's posture when joints value as $\left[0^{\circ} 0^{\circ} 0^{\circ} 0^{\circ} 0^{\circ} 0^{\circ}\right]$.

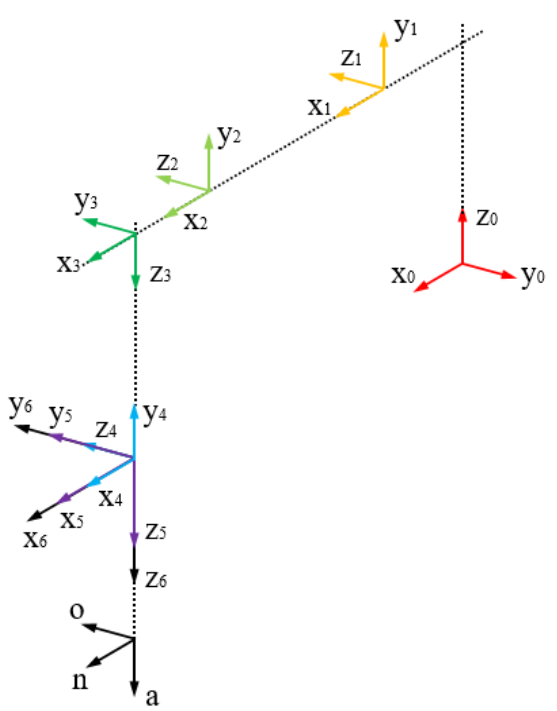

Figure 4: Reference frames representation of Nachi robot.

\begin{tabular}{|c|c|c|c|c|}
\hline $\boldsymbol{i}$ & $\boldsymbol{\theta}_{\boldsymbol{i}}$ & $\boldsymbol{d}_{\boldsymbol{i}}$ & $\boldsymbol{a}_{\boldsymbol{i}}$ & $\boldsymbol{a}_{\boldsymbol{i}}$ \\
\hline $\mathbf{1}$ & $\boldsymbol{\theta}_{\boldsymbol{1}}$ & 1070 & 340 & 90 \\
\hline $\mathbf{2}$ & $\boldsymbol{\theta}_{\mathbf{2}}$ & 0 & 910 & 0 \\
\hline $\mathbf{3}$ & $\boldsymbol{\theta}_{\mathbf{3}}$ & 0 & 200 & 90 \\
\hline $\mathbf{4}$ & $\boldsymbol{\theta}_{\mathbf{4}}$ & 1300 & 0 & -90 \\
\hline $\mathbf{5}$ & $\boldsymbol{\theta}_{\mathbf{5}}$ & 0 & 0 & 90 \\
\hline $\mathbf{6}$ & $\boldsymbol{\theta}_{\boldsymbol{6}}$ & 0 & 0 & 0 \\
\hline Tool & 0 & 235 & 0 & 0 \\
\hline
\end{tabular}

Table 1: D-H model parameters of Nachi Robot (SC300F-02).

\section{Influence of Joint Angle Error on End-Effector's Position Accuracy}

From the D-H model of Nachi robot, the center point of the robot's fixing plate relative to the robot base reference frame (Figure 5) can be described as the following equation:

$$
P=\left[\begin{array}{c}
x \\
y \\
z \\
1
\end{array}\right]=A_{1} A_{2} A_{3} A_{4} A_{5} A_{6} \cdot \text { Tool }
$$




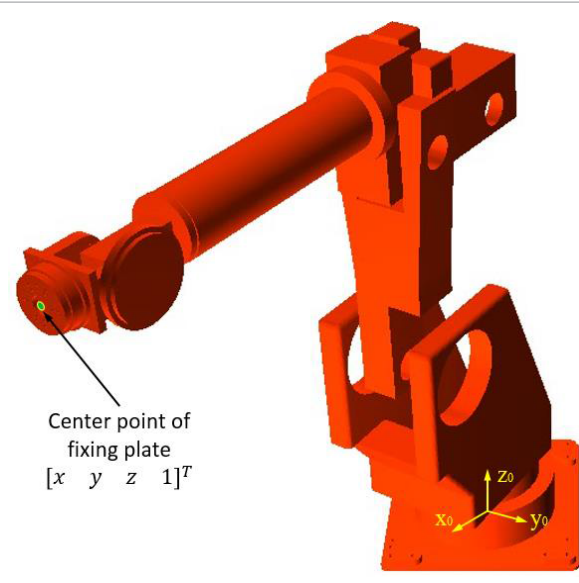

Figure 5: Center point of the robot's fixing plate relative to the robot base frame.

$$
\begin{aligned}
& \boldsymbol{A}_{i}=\left[\begin{array}{cccc}
C \theta_{i} & -S \theta_{i} C \alpha_{i} & S \theta_{i} S \alpha_{i} & a_{i} C \theta_{i} \\
S \theta_{i} & C \theta_{i} C \alpha_{i} & -C \theta_{i} S \alpha_{i} & a_{i} S \theta_{i} \\
0 & S \alpha_{i} & C \alpha_{i} & d_{i} \\
0 & 0 & 0 & 1
\end{array}\right] \\
& \text { Tool }=\left[\begin{array}{l}
0 \\
0 \\
l \\
1
\end{array}\right]
\end{aligned}
$$

In actual use, the kinematic parameters of the robot are normally different from the designed due to variety reasons, these errors come from the manufacturing, assembling, installation, sensors and even the temperature changing. Finally, these factors will lead to the position error of the end effector. Because the serial system structure of industrial robot, the error on each joint could be coupling and accumulate with each other. For the error on each joint, the ability to influence the final position error of the end effector is very different as well. Thus, a robot position error model can be created to analyze the sensitivity of each joint with error as following:

$$
\begin{aligned}
& \boldsymbol{T o o l}_{r}=\left[\begin{array}{c}
0 \\
0 \\
l+\delta l \\
1
\end{array}\right] \\
& \boldsymbol{P}_{r}=\left[\begin{array}{c}
x+\delta x \\
y+\delta y \\
z+\delta z \\
1
\end{array}\right]=\boldsymbol{A}_{1 r} \boldsymbol{A}_{2 r} \boldsymbol{A}_{3 r} \boldsymbol{A}_{4 r} \boldsymbol{A}_{5 r} \boldsymbol{A}_{6 r} \cdot \text { Tool }_{r} \\
& \Delta \boldsymbol{P}=\left|\boldsymbol{P}_{r} \boldsymbol{P}\right|=\sqrt{(\delta x)^{2}+(\delta y)^{2}+(\delta z)^{2}}
\end{aligned}
$$$$
\left[\begin{array}{llll}
C\left(\theta_{i}+\delta \theta_{i}\right) & -S\left(\theta_{i}+\delta \theta_{i}\right) C\left(\alpha_{i}+\delta \alpha_{i}\right) & S\left(\theta_{i}+\delta \theta_{i}\right) S\left(\alpha_{i}+\delta \alpha_{i}\right) & \left(a_{i}+\delta a_{i}\right) C\left(\theta_{i}+\delta \theta_{i}\right)
\end{array}\right]
$$$$
A_{i r}=\left[\begin{array}{cccc}
S\left(\theta_{i}+\delta \theta_{i}\right) & C\left(\theta_{i}+\delta \theta_{i}\right) C\left(\alpha_{i}+\delta \alpha_{i}\right) & -C\left(\theta_{i}+\delta \theta_{i}\right) S\left(\alpha_{i}+\delta \alpha_{i}\right) & \left(a_{i}+\delta a_{i}\right) S\left(\theta_{i}+\delta \theta_{i}\right) \\
0 & S\left(\alpha_{i}+\delta \alpha_{i}\right) & C\left(\alpha_{i}+\delta \alpha_{i}\right) & d_{i}+\delta d_{i} \\
0 & 0 & 0
\end{array}\right]
$$

$A_{i r}$ and Tool $_{r}$ are the transformation matrixes with kinematic parameters' error, $P_{r}$ is the center point of the robot's fixing plate when considering the kinematic parameters' error of $\mathrm{D}-\mathrm{H}$ model, $\Delta P$ is the position difference between the theory coordinate value and coordinate value with parameters' error.

Based on the equations of robot position error model, a D-H model parameter error analysis simulation system can be programmed with
Python, the flow chart of this simulation analysis system as shown in Figure 6.

For the error on each joint, the influence on final position error of the end effector is varied at different position and orientation. In order to study the difference of these influence, the control variable method and unified error input method has been adopted. Set the joint value as $\left[-90^{\circ} 70^{\circ}-20^{\circ} 0^{\circ}-50^{\circ} 90^{\circ}\right]$, this is a typical position and orientation of robot for deposition or machining process, as shown in Figure 7.

Use this as the robot's basic position and orientation, only rotate one joint at one time within its rotation range and keep other joints fixed, assume there is a joint angle error which value is $0.001^{\circ}$, calculate the coordinate error at every $1^{\circ}$ angle changing, apply this error on each joint respectively, thus a Figure with six error curves according to each joint can be obtained. Repeat this process for other joints, similar figures of error curves can be obtained, as shown in Figure 8 .

When joint 1 rotates from $0^{\circ}$ to $360^{\circ}$, meanwhile, other joints are fixed. Apply the $0.01^{\circ}$ joint angle error on each joint respectively, the resulting end effector error distribution as shown in Figure 8a. The Figure shows that when only joint 1 rotates, the end effector error caused by angle error on different joints are constant, these values don't change with the changing of position and orientation of joint 1 . For the

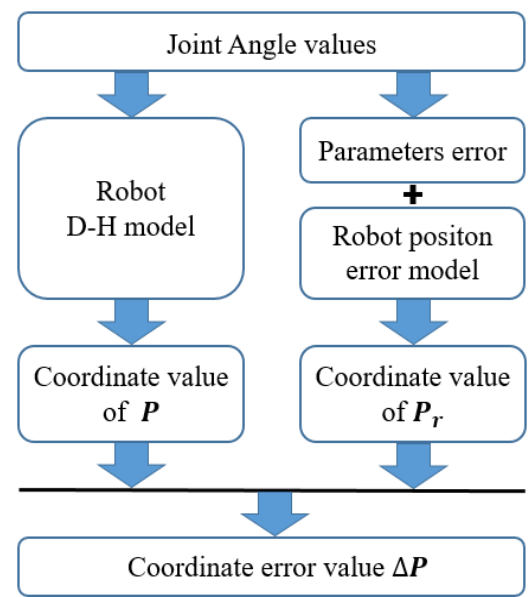

Figure 6: Flow chart of D-H model parameter error analysis simulation system.

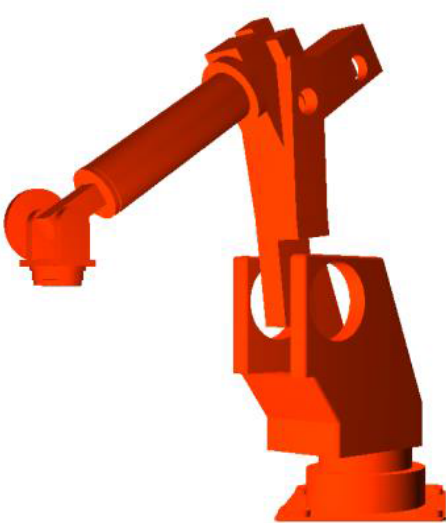

Figure 7: Typical position and orientation of robotic deposition or machining process. 
influence on final position error of different joint error, the effect of weights sorted descend as $1,3,2,5,4,6$.

When joint 2 rotates from $0^{\circ}$ to $150^{\circ}$, meanwhile, other joints are fixed. Apply the 0.01 joint angle error on each joint respectively, the resulting end effector error distribution as shown in Figure 8b. The Figure shows that when only joint 2 rotates, the end effector error caused by angle error on joint 1 is varied and reach its maximum at the middle value of the joint 2 rotation angle, the end effector error caused by angle error on other joints are constant, these values don't change with the changing of position and orientation of joint 2. For the influence on final position error of different joint error, the effect of weights sorted descend as $3,2,1,5,4,6$.

When joint 3 rotates from $-60^{\circ}$ to $30^{\circ}$, meanwhile, other joints are fixed. Apply the $0.01^{\circ}$ joint angle error on each joint respectively, the resulting end effector error distribution as shown in Figure 8c. The figure shows that when only joint 3 rotates, the end effector error caused by angle error on joint 1,2 are varied, the end effector error caused by angle error on other joints are constant, these values don't change with the changing of position and orientation of joint 3 . For the influence on final position error of different joint error, the effect of weights sorted descend as $1,2,3,5,4,6$.

When joint 4 rotates from $0^{\circ}$ to $360^{\circ}$, meanwhile, other joints are fixed. Apply the $0.01^{\circ}$ joint angle error on each joint respectively, the resulting end effector error distribution as shown in Figure $8 \mathrm{~d}$. The Figure shows that when only joint 4 rotates, the end effector error caused by angle error on joint 1,2,3 are varied, the end effector error caused by angle error on other joints are constant, these values don't change with the changing of position and orientation of joint 4 . For the influence on final position error of different joint error, the effect of weights sorted descend as 1, 2, 3, 5, 4, 6 .

When joint 5 rotates from $-120^{\circ}$ to $120^{\circ}$, meanwhile, other joints are fixed. Apply the $0.01^{\circ}$ joint angle error on each joint respectively, the resulting end effector error distribution as shown in Figure 8e. The Figure shows that when only joint 5 rotates, the end effector error caused by angle error on joint 1,2, 3, 4 are varied, the end effector error caused by angle error on other joints are constant, these values don't change with the changing of position and orientation of joint 5 . For the influence on final position error of different joint error, the effect of weights sorted descend as $1,2,3,5,4,6$.

When joint 6 rotates from $0^{\circ}$ to $360^{\circ}$, meanwhile, other joints are fixed. Apply the $0.01^{\circ}$ joint angle error on each joint respectively, the resulting end effector error distribution as shown in Figure $8 \mathrm{f}$. The Figure shows that when only joint 6 rotates, the end effector error caused by angle error on different joints are constant, these values don't change with the changing of position and orientation of joint 6 . For the influence on final position error of different joint error, the effect of weights sorted descend as $1,3,2,5,4,6$.

Sum up all these position errors together and calculate the average position error caused by each joint respectively, the results as shown in Table 2. As can be seen from the data in Table 2, even a tiny joint error can lead to a significant end effector position error. The sensitivity of joint error influence on end effector position error is different, for

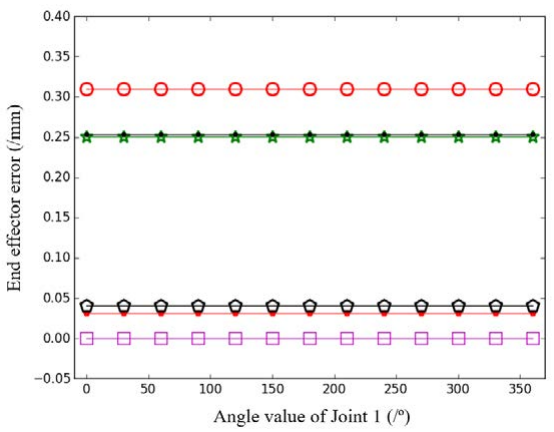

(a) Joint 1 rotate and other joints fixed

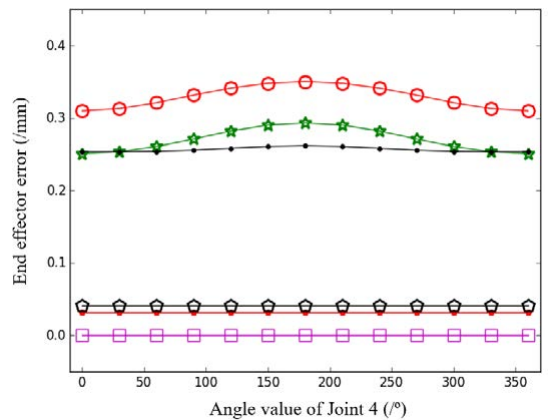

(d) Joint 4 rotate and other joints fixed

O Joint 1 with unified error

O-O Joint 3 with unified error th Joint 4 with unified error

ט Joint 5 with unified error

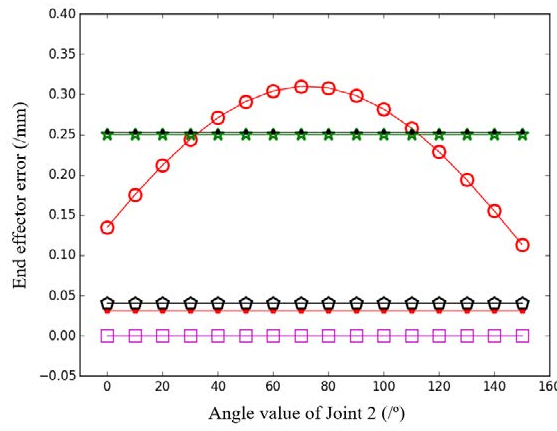

(b) Joint 2 rotate and other joints fixed

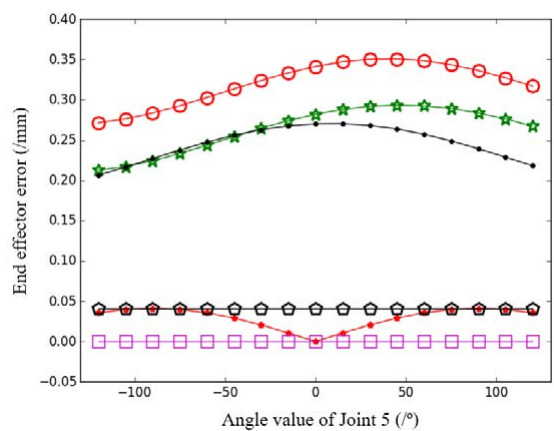

(e) Joint 5 rotate and other joints fixed

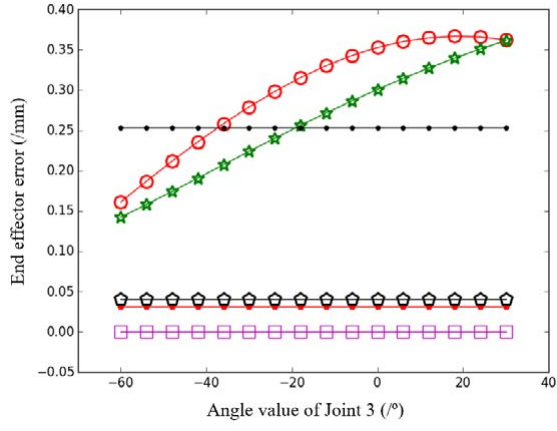

(c) Joint 3 rotate and other joints fixed

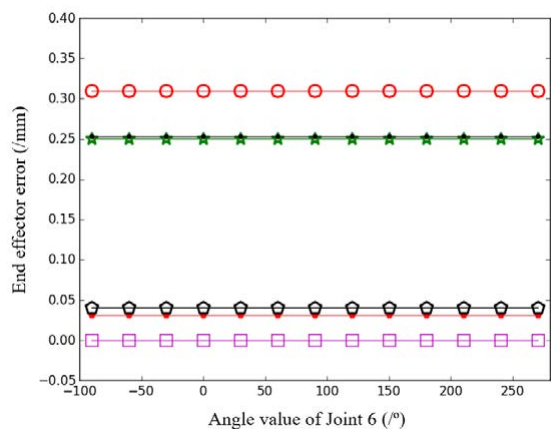

(f) Joint 6 rotate and other joints fixed

Figure 8: End effector error distribution when joints rotate with unified angle error (a-f). 


\begin{tabular}{|c|c|c|c|c|c|}
\hline & Joint $\mathbf{1}$ & Joint 2 & Joint 3 & Joint 4 & Joint $\mathbf{5}$ \\
\hline Average position error $\mathbf{( m m )}$ & 0.3010 & 0.2576 & 0.2524 & 0.0308 & 0.0410 \\
\hline Sensitivity rank & 6 & 5 & 4 & 2 \\
\hline
\end{tabular}

Table 2: Average position error (/mm) caused by each joint and joint error sensitivity rank.

the serial manipulator type industrial robot, the joint errors exist on arm joints have a more obvious effect on position error of end effector than the joint errors exist on wrist joints. For the total influence on final position error of different joint errors, the effect of weights sorted descend as $6,5,4,2,3,1$.

In order to increase the accuracy of an industrial robot, precisely manufactured parts and high-resolution sensors can be used to reduce the joint angle error, but adopt these expensive parts for the whole robot system will make the cost surge. The analysis of joint error sensitivity can be helpful for making a decision of balancing the cost and accuracy. Take this Nachi robot as an example, utilize high-performance parts and sensors on joint 1 will improve the system accuracy mostly.

\section{Industrial Robot Trajectory Accuracy Mapping}

Normally, the users pay attention to movement accuracy when robot perform certain trajectory, and simply believe that the more accurate of the robot system, the better result will be obtained. But, it is important to notice that the kinematic parameters error cannot be eliminated from the robot system completely, even after calibration, these errors still exist and will be varied during running, so the conventional error compensation method is not a "once and for all" solution. For a certain working path, it can be performed at multiple positions and orientations within robot working envelope. Based on the robot kinematic and joint sensitivity analysis, a visualized evaluation map can be obtained to describe the accuracy difference of a specific trajectory at different positions and orientations. This method can help the user to find the best position and orientation to perform a specific working path, it can also make the maximum usage of current accuracy ability of a specific robot rather than blindly pursuit higher accuracy.

Any working path performed by the robot is composed of angle changing in joints domain, the angle changing is related with the trajectory itself, as well as with its location and orientation. When an error is present, each joint has different sensitivity on affecting the position error of end effector. Thus, a trajectory accuracy evaluation function of two points can be created as the following:

$$
E=\sum_{i=1}^{6} w_{i} \Delta \theta_{i}=\left[\begin{array}{llllll}
w_{1} & w_{2} & w_{3} & w_{4} & w_{5} & w_{6}
\end{array}\right]\left[\begin{array}{c}
\Delta \theta_{1} \\
\Delta \theta_{2} \\
\Delta \theta_{3} \\
\Delta \theta_{4} \\
\Delta \theta_{5} \\
\Delta \theta_{6}
\end{array}\right]
$$

$w_{i}$ is the effect weight of different joints influence on position of end effector, $\Delta \theta i$ is the robot joint angle changing of between two point.

For example, make the end effector move a $50 \mathrm{~mm}$ straight line along the $y$-axis from negative to positive in robot system coordinate, there are multiple positions available to conduct this task within robot working envelope, as shown in Figure 9.

Apply the trajectory accuracy evaluation method for this task, separate working area into small testing patches $(50 \mathrm{~mm} \times 50 \mathrm{~mm})$ within the $\mathrm{x}$ range is from -700 to 700 , $y$ range is from -1200 to $-2000, \mathrm{z}$ takes $800,1200,1600$, and 2000 , respectively, in robot system

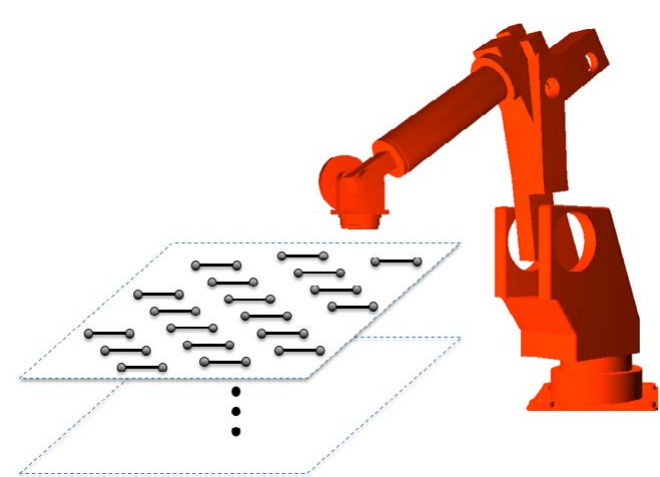

Figure 9: Multiple positions choice for robot conduct a specific trajectory.

coordinate. Based in on the current angle error value of Nachi Robot (SC300F-02) which obtained from the calibration process, the robot trajectory accuracy evaluation mapping results for a $50 \mathrm{~mm}$ straight line in these laminated 2D working areas are as shown in Figure 10.

As can be seen from Figure 10, for same height, which means $\mathrm{z}$ value is constant, each patch has different accuracy evaluation value for a specific trajectory, the less evaluation value of patch is, the better accuracy can be obtained at this position. The patch surrounded by yellow rectangle shape indicate the best position to perform this task. The best position is varied along with changing of $\mathrm{z}$ value.

In order to verify the correctness of accuracy evaluation, the drawing experiments have carried out.

Employ the parameter settings the in Figure 10a as an example, set up the position of the working table and white paper with the grid in the robot working envelope, as shown in Figure 11.

According to the accuracy evaluation results shown in Figure 10a, let the robot draw a $50 \mathrm{~mm}$ straight line in the patch with the best accuracy and in the patch with worst accuracy respectively.

Repeat this process for 10 times and record the measurement data as shown in Figure 12.

As can be seen from Figure 12, the average measurement value at the best accuracy position and the worst accuracy position are 50.01 $\mathrm{mm}$ and $50.14 \mathrm{~mm}$, the standard deviation of two sets of data are 0.074 and 0.107 , respectively. The difference between two standard deviations is $31 \%$.

The accuracy evaluation of the best accuracy position and the worst accuracy position with the parameter settings in Figure 10a are 2.65 and 3.74, the difference between two accuracy evaluation values is $29 \%$, which is close to the difference of standard deviations of experiments value. Thus the experiments result shows that the accuracy evaluation value could reflect the trajectory accuracy difference within robot working envelope at different positions.

It is easy to notice that in Figure 9, all the directions of the straight lines are along the y-axis of robot system coordinate, it is also the common direction when the users assign a working path for the robot. Obviously, this straight line can be drawn in multiple directions from 
Citation: Wang Z, Liu R, Sparks T, Chen X, Liou F (2018) Industrial Robot Trajectory Accuracy Evaluation Maps for Hybrid Manufacturing Process Based on Joint Angle Error Analysis. Adv Robot Autom 7: 183. doi: 10.4172/2168-9695.1000183

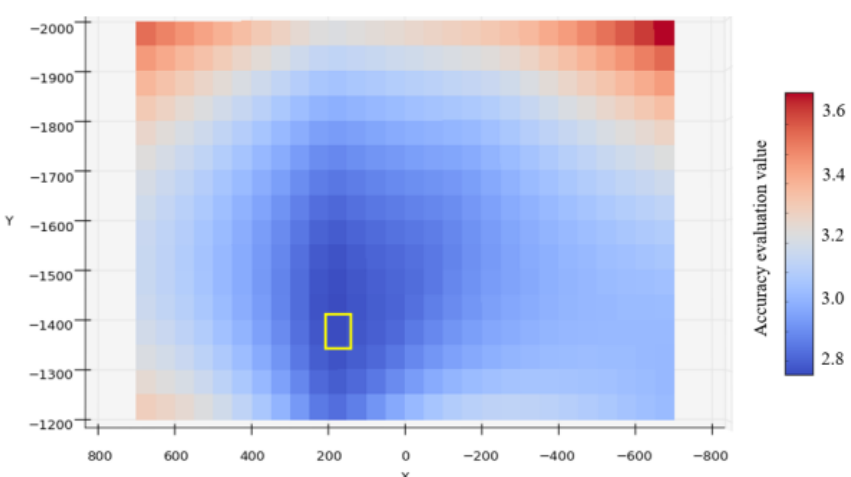

(a) $\mathrm{X}(-700,700), \mathrm{Y}(-1200,1200), \mathrm{Z}=800$

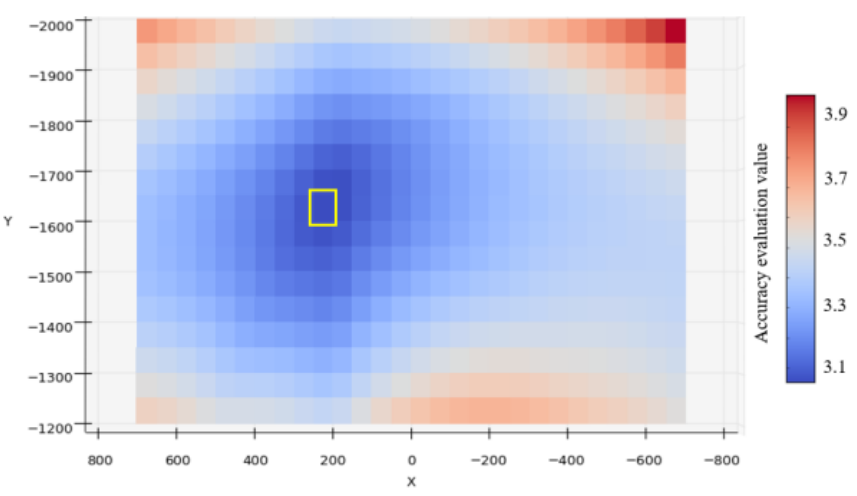

(c) $\mathrm{X}(-700,700), \mathrm{Y}(-1200,1200), \mathrm{Z}=1600$

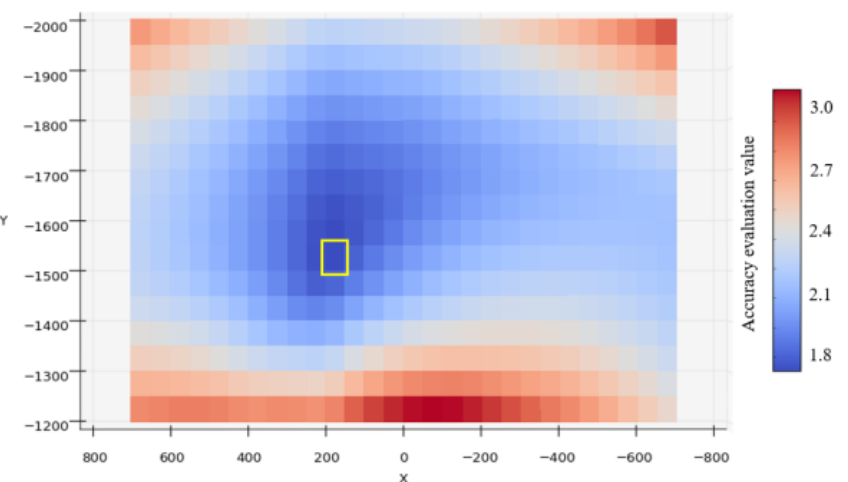

(b) $\mathrm{X}(-700,700), \mathrm{Y}(-1200,1200), \mathrm{Z}=1200$

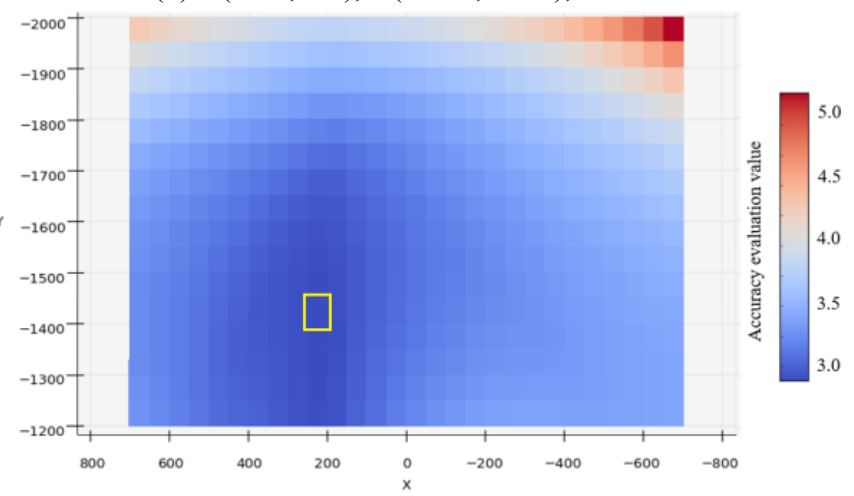

(d) $\mathrm{X}(-700,700), \mathrm{Y}(-1200,1200), \mathrm{Z}=2000$

Figure 10: Trajectory accuracy evaluation mapping result for a straight line in the 2D working area (a-d).

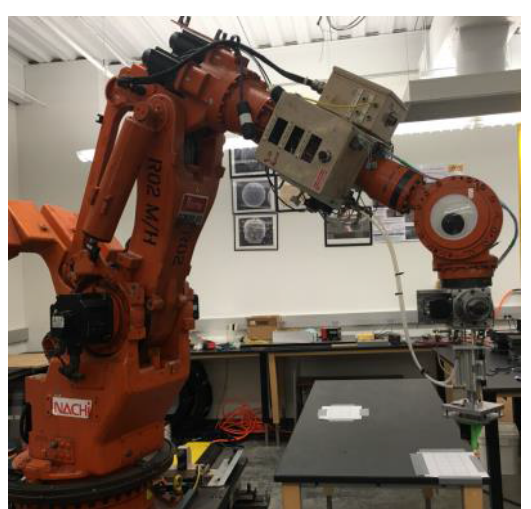

Figure 11: Experiments set up for accuracy evaluation of robot drawing straight line.

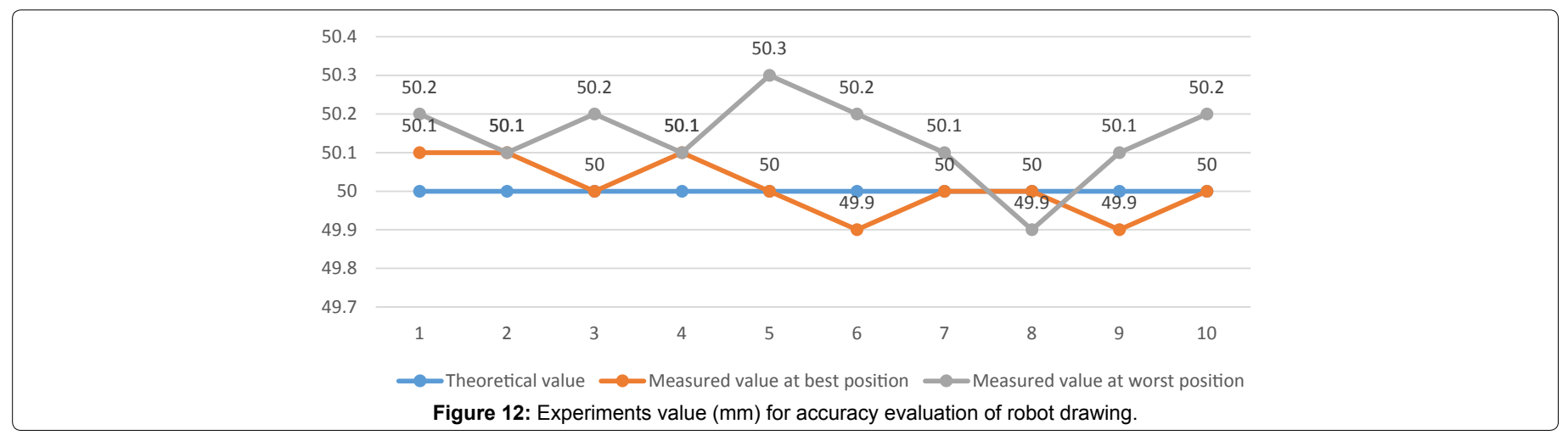


the same start point, and the different direction will cause different joint angle changing, as shown in Figure 13.

Apply the trajectory accuracy evaluation method for this situation, set the angle changing $30^{\circ}$ every position from $0^{\circ}$ to $360^{\circ}$, the start point is $(200,-1500,1200)$ in robot system coordinate, the length of the line is $50 \mathrm{~mm}$. The robot trajectory accuracy evaluation results for these lines toward different directions as shown in Figure 14.

The $50 \mathrm{~mm}$ straight line start from the same point toward different directions have different accuracy evaluation value, the color range from red to green indicates the accuracy evaluation value as descending, the less evaluation value of line is, the better accuracy can be obtained at this orientation. The lowest accuracy evaluation value is plotted as the thicker green arrow, it represents the best orientation to perform this task.

In order to observe the affection of orientation on trajectory accuracy in the working envelope of robot, separate working area into small testing patches $(120 \mathrm{~mm} \times 120 \mathrm{~mm})$ within the $\mathrm{x}$ range is from -600 to 600 , $y$ range is from -1200 to -1800 , $z$ takes $800,1200,1600$, and 2000 , respectively, in robot system coordinate. Then apply the same analysis process to these multiple centers, the robot trajectory accuracy evaluation mapping results for $50 \mathrm{~mm}$ straight line starts from the same point towards different directions in laminated $2 \mathrm{D}$ working areas are as shown in Figure 15.

As can be seen from Figure 15, at the same height, which means $\mathrm{z}$ value is constant, the best orientation for move a straight line are varied in different regions. For the same $\mathrm{x}, \mathrm{y}$ coordinates, the best orientation could be changed according to the changing of $\mathrm{z}$ value, as the red rectangle bounded area shown in these Figures. Thus, the best position and orientation in 3D working envelope to perform a certain working path can be found by taking enough accuracy evaluation calculation.

\section{Simulation: Trajectory Accuracy Mapping of a Robotic Hybrid Manufacturing Working Path}

The zigzag path is a typical trajectory for robotic hybrid manufacturing as shown in Figure 16. One layer of this kind path could work for machining or milling process, multiple layers of that could be used as a deposition working path.

The simulation will take this zigzag path as an example, use the above-discussed trajectory accuracy mapping method to find the best position and orientation to conduct this task within Nachi Robot's (SC300F-02) working envelope (Figure 17).

In order to study how zigzag trajectory's position and orientation affect its accuracy in the working envelope of the robot, the trajectory's accuracy evaluation value should be calculated at the different position while with different orientation within the robot working envelope.

Firstly, separate working volume into small testing cube area $(50 \mathrm{~mm} \times 50 \mathrm{~mm} \times 50 \mathrm{~mm})$ within the in robot system coordinate. Specifically, $\mathrm{x}$ range is from -500 to 500 , $\mathrm{y}$ range is from -1200 to -1800 ,

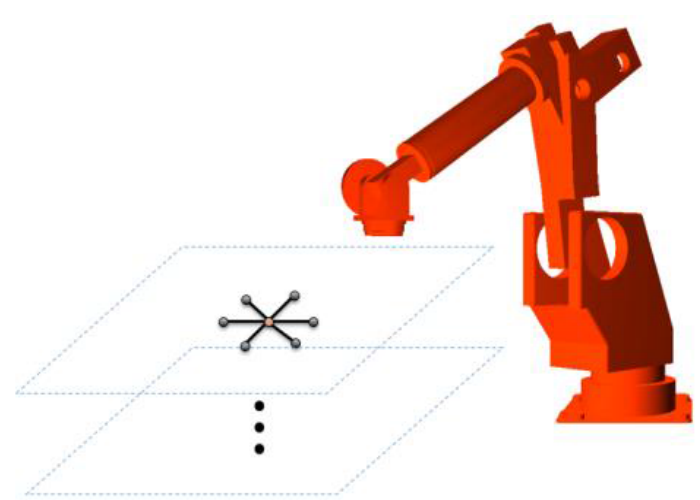

Figure 13: Multiple directions of the same length paths.

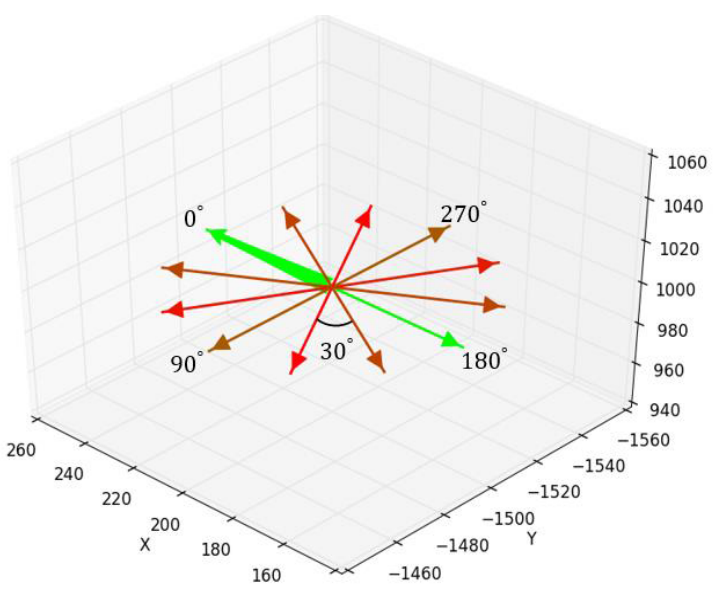

Figure 14: Trajectory accuracy evaluation mapping result for straight line towards different directions. 
Citation: Wang Z, Liu R, Sparks T, Chen X, Liou F (2018) Industrial Robot Trajectory Accuracy Evaluation Maps for Hybrid Manufacturing Process Based on Joint Angle Error Analysis. Adv Robot Autom 7: 183. doi: 10.4172/2168-9695.1000183

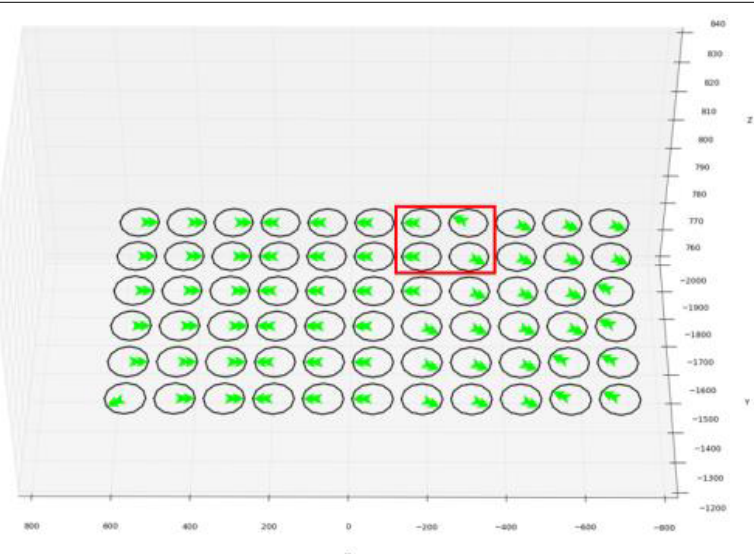

(a) $\mathrm{X}(-700,700), \mathrm{Y}(-1200,1200), \mathrm{Z}=800$

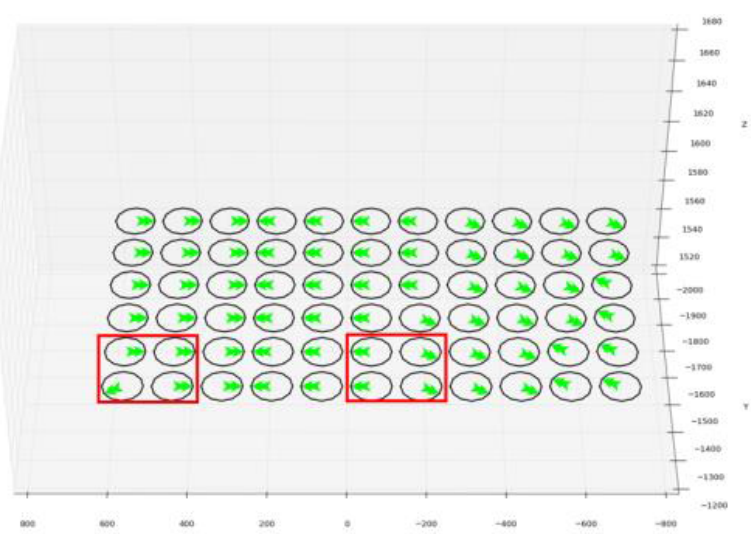

(c) $\mathrm{X}(-700,700), \mathrm{Y}(-1200,1200), \mathrm{Z}=1600$

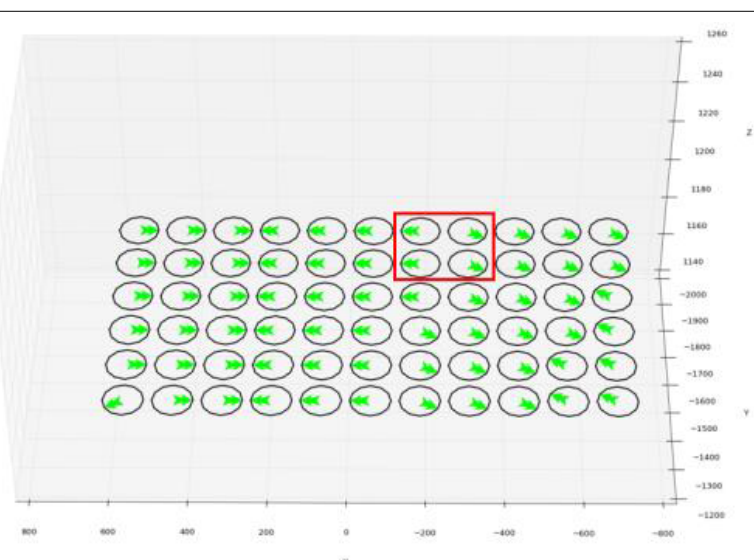

(b) $\mathrm{X}(-700,700), \mathrm{Y}(-1200,1200), \mathrm{Z}=1200$

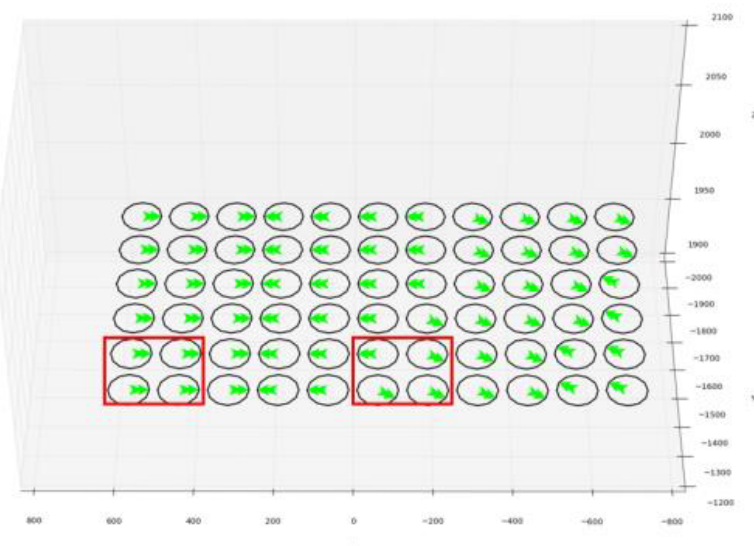

(d) $\mathrm{X}(-700,700), \mathrm{Y}(-1200,1200), \mathrm{Z}=2000$

Figure 15: Trajectory accuracy evaluation mapping result for straight line orientation analysis.

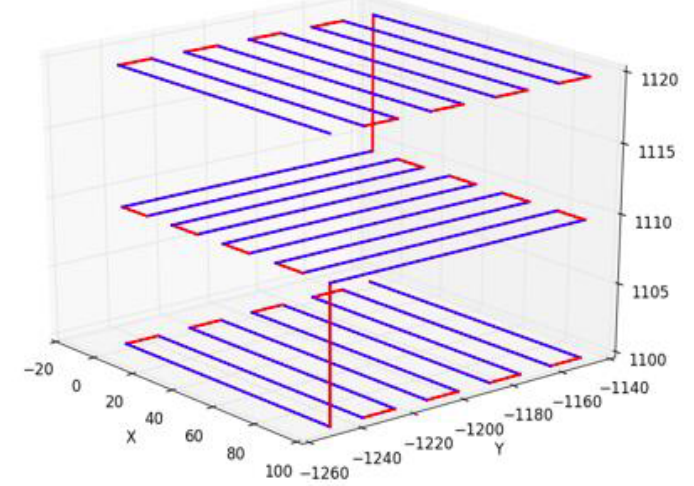

Figure 16: Zigzag path for hybrid manufacturing

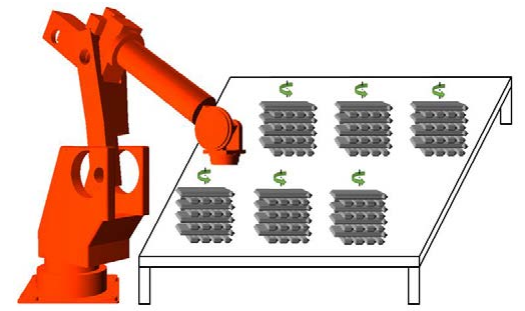

Figure 17: Multiple positions and orientation possibilities for a zigzag path.

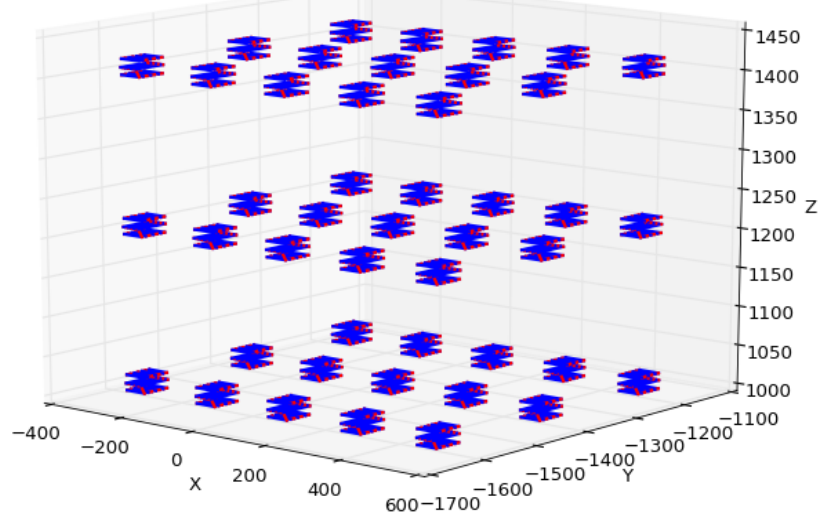

Figure 18: Trajectory testing cube within robot working envelope.

$\mathrm{z}$ range is from 800 to 1400 .Thus there are 45 testing cube areas within robot working envelope, as shown in Figure 18. The dimension of deposition zigzag path is $(10 \mathrm{~mm} \times 10 \mathrm{~mm} \times 1 \mathrm{~mm})$, the layer thickness is $0.1 \mathrm{~mm}$, track width is $2 \mathrm{~mm}$ and overlap is 0.3 .

Secondly, set the orientation angle for these trajectories: start from $\mathrm{x}$-axis positive direction, rotate about $\mathrm{z}$-axis counterclockwise, take the angle value as $0^{\circ}, 30^{\circ}, 60^{\circ}, 90^{\circ}, 120^{\circ}, 150^{\circ}, 180^{\circ}, 210^{\circ}, 240^{\circ}, 270^{\circ}, 300^{\circ}$, $330^{\circ}$, respectively. Then apply the trajectory accuracy mapping process 
Citation: Wang Z, Liu R, Sparks T, Chen X, Liou F (2018) Industrial Robot Trajectory Accuracy Evaluation Maps for Hybrid Manufacturing Process Based on Joint Angle Error Analysis. Adv Robot Autom 7: 183. doi: 10.4172/2168-9695.1000183

Page 9 of 12

to every line segments of the zigzag trajectories. Sum these up as the overall robot trajectory accuracy evaluation value of this trajectory, the results are respectively shown in Table 3. Plot the testing cube area with the normalized evaluation values in angle group, the trajectory accuracy mapping results for this task as shown in Figure 19.

As can be seen from Table 3 and Figure 19, the affection of position to the trajectory accuracy evaluation result is obvious. The accuracy evaluation result is also changing with the change of trajectory's orientation. Because the deposition angle between layers differs $90^{\circ}$, so the trajectory accuracy evaluation value in each axis directions is close, but it is still slightly different. The lower of the evaluation result is, the better accuracy can be obtained. Thus, the best position and orientation to perform this zigzag task is at center point of $[100,-1600,1000]$, and orientation angle is $90^{\circ}$.

For the laser metal deposition process, the track width is an important parameter to ensure the deposition quality, it will affect the gap distance between the melting pools, thereby influence the dimension accuracy and density of the deposited parts (Figure 20).

Usually, the diameter of the laser spot is $2 \mathrm{~mm}$, the overlap is chosen as $30 \%$, thereby the theoretical track width should be $1.4 \mathrm{~mm}$. But in actual, due to the motion error of moving system, a $10 \%$ tolerance of the theoretical value is acceptable, as shown in Figure 21.

\begin{tabular}{|c|c|c|c|c|c|c|c|c|c|c|c|c|}
\hline \multirow{2}{*}{$\begin{array}{c}\text { Position } \\
\text { No. }\end{array}$} & \multicolumn{12}{|c|}{ Rotation angle $\left({ }^{\circ}\right)$} \\
\hline & 0 & 30 & 60 & 90 & 120 & 150 & 180 & 210 & 240 & 270 & 300 & 330 \\
\hline 1 & 513.73 & 544.44 & 600.52 & 514.68 & 543.87 & 598.28 & 513.70 & 54450 & 600.83 & 514.97 & 544.02 & 598.26 \\
\hline 2 & 449.73 & 486.91 & 537.52 & 450.33 & 486.04 & 535.57 & 449.73 & 486.90 & 537.82 & 450.53 & 486.15 & 535.58 \\
\hline 3 & 418.42 & 458.35 & 504.35 & 418.92 & 457.10 & 502.23 & 418.42 & 458.30 & 504.41 & 419.04 & 457.21 & 502.23 \\
\hline 4 & 482.79 & 591.78 & 598.75 & 483.24 & 590.82 & 596.77 & 482.75 & 591.80 & 599.02 & 483.51 & 590.90 & 596.81 \\
\hline 5 & 415.23 & 52059 & 534.87 & 415.44 & 519.39 & 533.27 & 415.23 & 52059 & 535.16 & 415.59 & 519.50 & 533.27 \\
\hline 6 & 382.97 & 484.43 & 499.07 & 383.08 & 482.87 & 497.38 & 382.97 & 484.40 & 499.19 & 383.19 & 482.98 & 497.37 \\
\hline 7 & 494.84 & 610.41 & 571.85 & 494.79 & 608.92 & 570.37 & 494.81 & 610.63 & 572.11 & 494.96 & 608.99 & 570.40 \\
\hline 8 & 425.68 & 538.09 & 514.81 & 425.50 & 536.55 & 513.60 & 425.68 & 538.09 & 515.07 & 425.61 & 536.66 & 513.60 \\
\hline 9 & 386.03 & 500.67 & 483.25 & 385.73 & 498.75 & 481.91 & 386.03 & 500.61 & 483.36 & 385.84 & 498.86 & 481.91 \\
\hline 10 & 532.61 & 599.25 & 522.45 & 532.12 & 597.21 & 521.56 & 532.58 & 59951 & 522.70 & 532.21 & 597.30 & 521.58 \\
\hline 11 & 461.10 & 539.73 & 479.53 & 460.51 & 537.84 & 478.68 & 461.10 & 539.98 & 479.68 & 460.62 & 537.95 & 478.68 \\
\hline 12 & 421.69 & 506.31 & 458.00 & 420.98 & 503.98 & 457.02 & 421.69 & 506.20 & 458.11 & 421.09 & 504,08 & 457.02 \\
\hline 13 & 542.77 & 571.76 & 466.45 & 541.87 & 570.01 & 466.02 & 542.75 & 572.02 & 466.58 & 541.97 & 570.03 & 466.04 \\
\hline 14 & 482.07 & 527.45 & 434.70 & 481.07 & 525.64 & 434.22 & 482.07 & 527.68 & 434.82 & 481.19 & 525.64 & 434.22 \\
\hline 15 & 447.82 & 503.16 & 427.43 & 446.67 & 500,73 & 426.81 & 447.81 & 503.05 & 427.54 & 446.78 & 500,73 & 426.81 \\
\hline 16 & 517.89 & 547.34 & 608.58 & 518.73 & 546.65 & 606.57 & 518.11 & 54756 & 609.16 & 519.30 & 547.09 & 606.55 \\
\hline 17 & 448.80 & 48057 & 534.86 & 449.32 & 479.67 & 533.27 & 448.99 & 480.77 & 535.31 & 449.67 & 479.97 & 533.07 \\
\hline 18 & 418.38 & 452.09 & 498.91 & 418.78 & 450.75 & 496.95 & 418.50 & 452.09 & 498.99 & 419.04 & 451.01 & 496.78 \\
\hline 19 & 485.20 & 598.96 & 609.26 & 485.57 & 597.84 & 607.53 & 485.41 & 599.17 & 609.82 & 486.13 & 598.18 & 607.32 \\
\hline 20 & 409.20 & 516.04 & 532.82 & 409.35 & 514.81 & 531.54 & 409.39 & 516.23 & 533.27 & 409.67 & 515.11 & 531.34 \\
\hline 21 & 383.19 & 477.86 & 493.14 & 383.22 & 476.22 & 491.73 & 383.33 & 477.38 & 493.42 & 383.49 & 476.49 & 491.56 \\
\hline 22 & 497.96 & 61757 & 580.39 & 497.84 & 616.03 & 579.16 & 498.16 & 618.07 & 580.94 & 498.28 & 616.37 & 578.95 \\
\hline 23 & 418.31 & 534.70 & 512.48 & 418.07 & 533.13 & 511.54 & 418.50 & 534.89 & 512.88 & 418.39 & 533.45 & 511.34 \\
\hline 24 & 381.87 & 493.63 & 477.77 & 381.52 & 491.64 & 476.70 & 382.01 & 493.63 & 478.05 & 381.79 & 491.92 & 476.54 \\
\hline 25 & 533.36 & 603.66 & 523.84 & 532.78 & 601.76 & 523.20 & 533.56 & 604.20 & 524.36 & 533.13 & 601.86 & 523.00 \\
\hline 26 & 453.99 & 535.06 & 476.23 & 453.35 & 533.18 & 475.64 & 454.17 & 535.32 & 476.55 & 453.67 & 533.44 & 475.46 \\
\hline 27 & 414.20 & 498.61 & 453.88 & 413.41 & 496.24 & 453.16 & 414.31 & 498.62 & 454.16 & 413.68 & 496.51 & 453.00 \\
\hline 28 & 538.97 & 570.86 & 460.17 & 537.98 & 569.38 & 459.97 & 539.15 & 571.39 & 460.52 & 538.33 & 569.20 & 459.79 \\
\hline 29 & 475.20 & 5210.8 & 430.48 & 474.15 & 519.48 & 430.25 & 475.37 & 521.38 & 430.79 & 474.47 & 519.32 & 430.08 \\
\hline 30 & 439.15 & 494.75 & 424.61 & 437.89 & 492.47 & 424.24 & 439.21 & 494.78 & 424.89 & 438.16 & 492.35 & 424.09 \\
\hline 31 & 497.12 & 517.68 & 582.44 & 497.66 & 517.01 & 581.32 & 497.62 & 518.17 & 583.28 & 498.43 & 517.69 & 581.23 \\
\hline 32 & 437.95 & 462.20 & 516.80 & 438.26 & 461.20 & 515.52 & 438.26 & 462.42 & 517.11 & 438.74 & 461.67 & 515.24 \\
\hline 33 & 411.88 & 437.47 & 488.94 & 412.07 & 436.07 & 487.19 & 412.04 & 437.63 & 489.10 & 412.48 & 436.48 & 486.86 \\
\hline 34 & 455.38 & 569.26 & 586.35 & 455.56 & 568.22 & 585.46 & 455.88 & 569.75 & 587.21 & 456.33 & 568.85 & 584.96 \\
\hline 35 & 399.03 & 495.83 & 513.15 & 399.04 & 494.57 & 512.34 & 399.38 & 496.09 & 513.65 & 399.54 & 495.06 & 511.93 \\
\hline 36 & 377.59 & 461.69 & 482.91 & 377.44 & 460.01 & 481.81 & 377.77 & 461.85 & 483.27 & 377.86 & 460.43 & 481.47 \\
\hline 37 & 468.85 & 588.43 & 558.90 & 468.63 & 587.03 & 558.38 & 469.32 & 588.93 & 559.74 & 469.30 & 587.66 & 557.90 \\
\hline 38 & 398.21 & 513.68 & 493.72 & 397.88 & 512.10 & 493.21 & 398.56 & 513.91 & 494.24 & 398.39 & 512.61 & 492.82 \\
\hline 39 & 376.18 & 476.75 & 467.89 & 375.65 & 474.75 & 467.18 & 376.35 & 476.91 & 468.33 & 376.08 & 475.18 & 466.85 \\
\hline 40 & 503.44 & 578.48 & 504.20 & 502.82 & 577.28 & 504,04 & 503.87 & 579.30 & 504.89 & 503.44 & 577.41 & 503.60 \\
\hline 41 & 434.48 & 514.28 & 460.77 & 433.75 & 512.54 & 460.56 & 434.78 & 514.46 & 461.29 & 434.27 & 512.86 & 460.20 \\
\hline 42 & 403.45 & 482.23 & 444.92 & 402.52 & 479.88 & 444.52 & 403.61 & 482.39 & 445.36 & 402.95 & 480.30 & 444.21 \\
\hline 43 & 513.70 & 546.94 & 436.79 & 512.70 & 546.04 & 436.99 & 514.08 & 547.62 & 437.39 & 513.30 & 545.65 & 436.60 \\
\hline 44 & 455.29 & 50150 & 420.15 & 454.13 & 500.11 & 420.26 & 455.52 & 501.72 & 420.65 & 454.63 & 499.78 & 419.92 \\
\hline 45 & 426.86 & 480.38 & 416.91 & 425.49 & 478.27 & 416.84 & 427.02 & 48053 & 417.34 & 425.91 & 478.11 & 416.56 \\
\hline
\end{tabular}

Table 3: Zigzag trajectories accuracy evaluation value. 


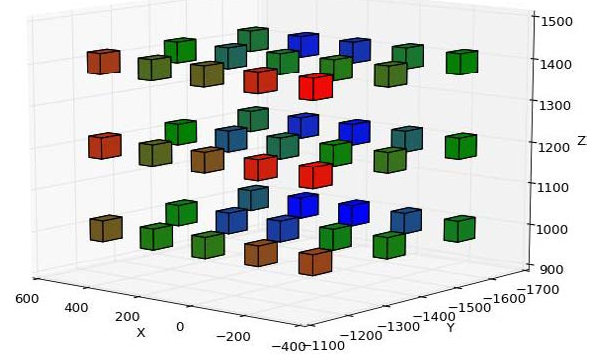

(a) Trajectory rotate angle equals $0^{\circ}$

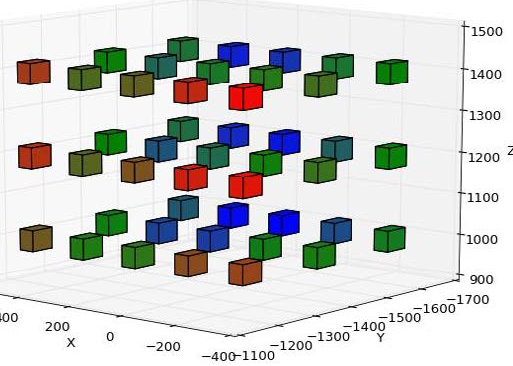

(d) Trajectory rotate angle equals $90^{\circ}$

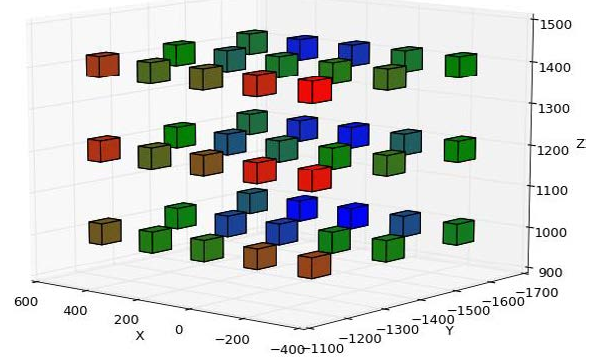

(g) Trajectory rotate angle equals $180^{\circ}$

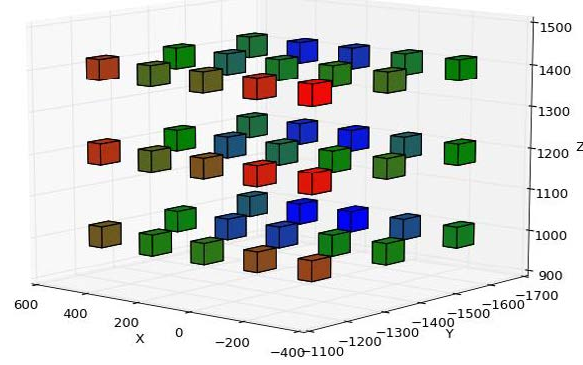

(j) Trajectory rotate angle equals $270^{\circ}$

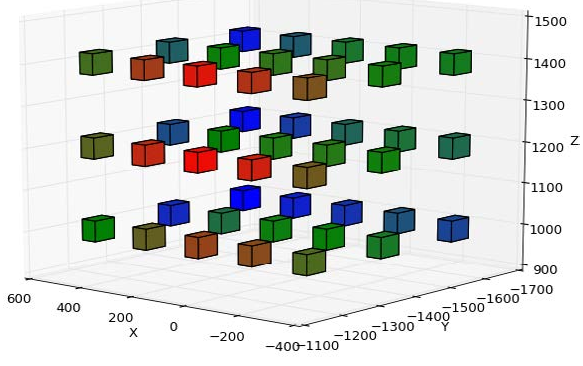

(b) Trajectory rotate angle equals $30^{\circ}$

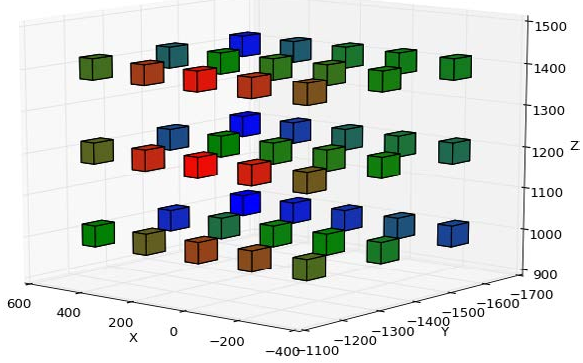

(e) Trajectory rotate angle equals $120^{\circ}$

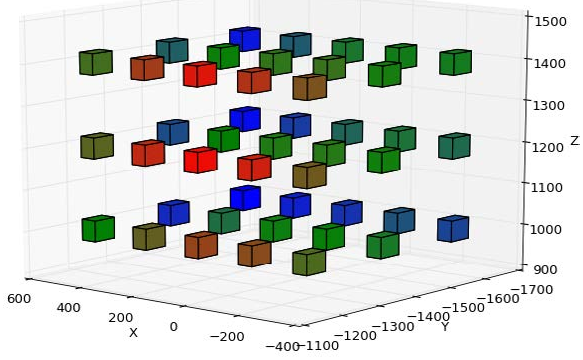

(h) Trajectory rotate angle equals $210^{\circ}$

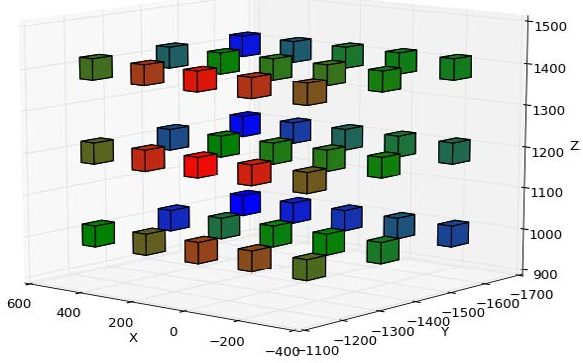

(k) Trajectory rotate angle equals $300^{\circ}$

Color legend of Normalized Value

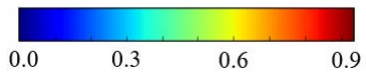

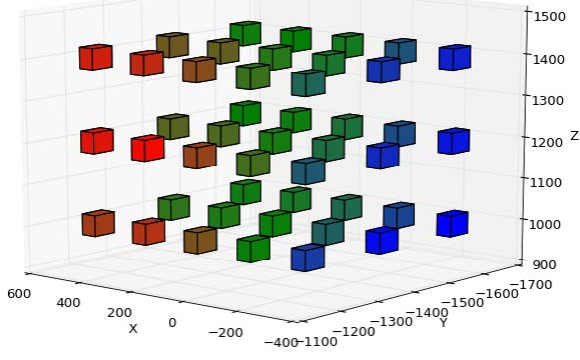

(c) Trajectory rotate angle equals $60^{\circ}$

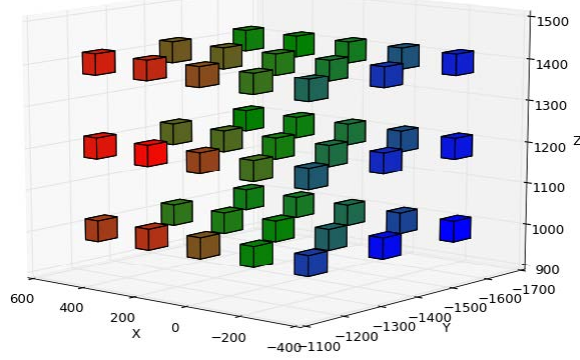

(f) Trajectory rotate angle equals $150^{\circ}$

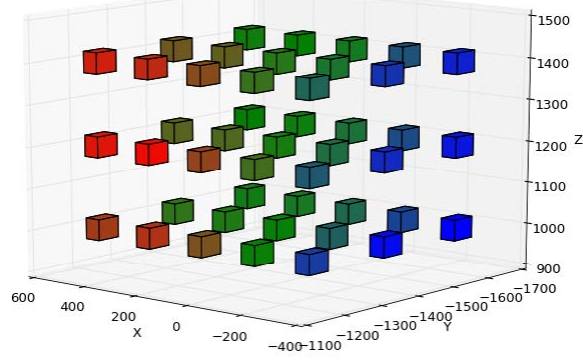

(i) Trajectory rotate angle equals $240^{\circ}$

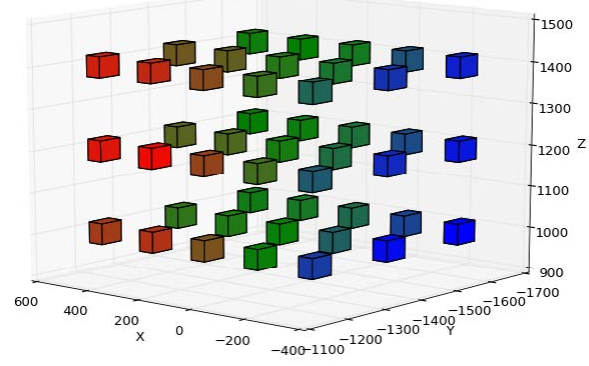

(1) Trajectory rotate angle equals $330^{\circ}$

Figure 19: Trajectory accuracy mapping results (cont.).

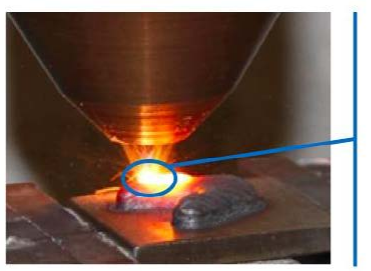

Figure 20: Schematic diagram of track width and melting in laser metal deposition.

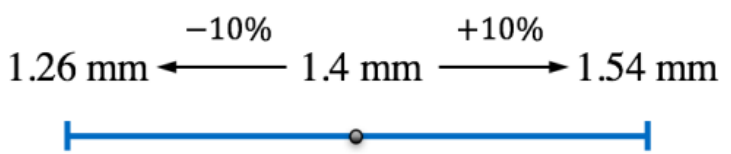

Acceptable range

Figure 21: Deposition track width tolerance. 


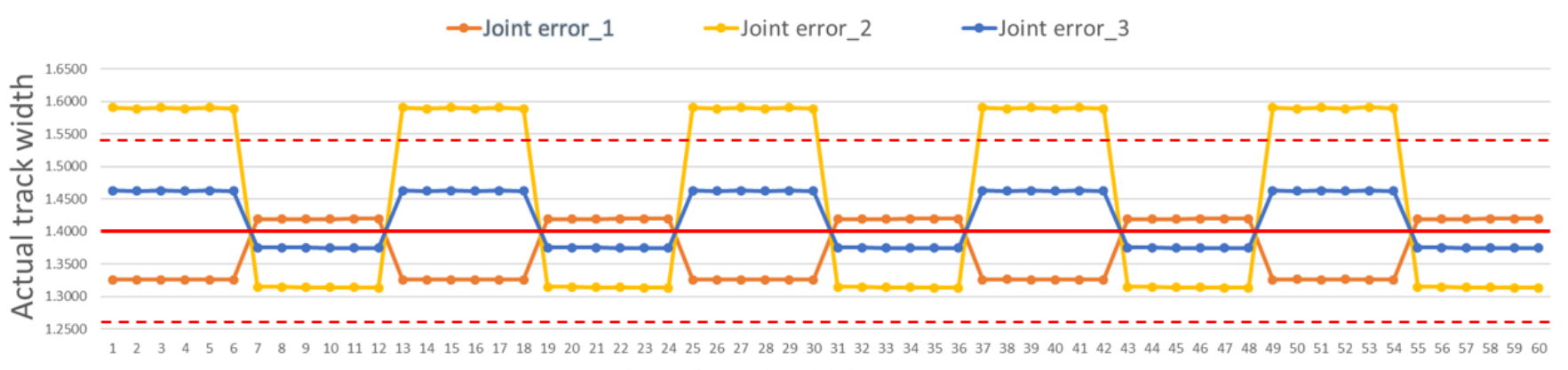

Index of track width segments

Figure 22: Actual track width according to different joint error.

With a group of known joint error value, the actual track width conduct by robot at the best position and orientation can be calculated through the robot kinematic. The joint error can be obtained by using many kinds of robot calibration method, like the laser tracker or machine vision. In order to discuss the affection of joint error on actual deposition track width, the joint error can be assumed with consideration of the robot's structure, wearing state, and its working environment. Three groups of joint error have been assumed as following: Joint error_1 $[0.011,-0.26,0.05,-0.01,-0.04,-0.01]$, Joint error_2 [0.023, $0.05,-0.29,0.38,-0.03,0.01]$, Joint error_3 [-0.013, 0.17 , $-0.09,0.15,0.04,0.01]$.

There are total of 60 track width segments in the deposition working path, the actual track width according to these three groups of joint error has been plotted as shown in Figure 22.

The red solid line is the theoretical value of track width, the two red dash lines are the lower and upper tolerance of this theoretical value. As can be seen from Figure 22, Joint error_2 exceed the upper tolerance, that means if robot's joints with this group of joint error value, it cannot satisfy the requirements of laser deposition task. In this case, the robot system is needed to be calibrated or applied with other compensation methods to improve its movement and position accuracy performance. For the other two groups of joint error, all of the track width value can fall into the acceptable tolerance range, but the distribution of deviation from the theoretical value is different. Joint error_3 could result lager actual track width values than Joint error_1, it is preferred for the actual additive process, because this provides more manufacturing allowance for the next step machining process in hybrid manufacturing.

\section{Conclusion}

The subject of this paper was to develop a new methodology for finding the best position and orientation to perform specific tasks based on the current robot system accuracy capability. Firstly, the D-H model of Nachi Robot (SC300F-02) was established. Since joint angle error affects the end effector position accuracy greatly, a robot position error model was created to analyze the sensitivity of each joint with angle error. It reveals that even the same joint angle error could have a different weight of affection when it appears on the different joint. Thus, a new evaluation formulation was established for mapping the trajectory accuracy within the robot's working volumetric. With a group of known joint error, the influence of different position and orientation on the movement accuracy of end effector was discussed. Finally, the simulation process takes a laser deposition zigzag working path as an example to validate the effectiveness of the proposed methodology, it also can be used as a criterion for checking the current joint error of robot system whether can satisfy a specific manufacturing tolerance or not. In addition, this method not only benefits the application of using the robot in the hybrid manufacturing process, it also important for improving robot operation accuracy performance in other area and optimizing the cost design of the industrial robot.

\section{Acknowledgements}

This research was sponsored by Laser Aided Manufacturing Processes (LAMP) Laboratory at Missouri University of Science and Technology (Missouri S\&T). Their support is greatly appreciated. The author would also like to thank all the people attached directly or indirectly to the project.

\section{References}

1. Summers M (2005) Robot capability test and development of industrial robot positioning system for the aerospace industry. SAE Technical Paper.

2. Klimchik A, Furet B, Caro S, Pashkevich A (2015) Identification of the manipulator stiffness model parameters in industrial environment. Mechanism and Machine Theory 90: 1-22.

3. Veitschegger W, Wu CH (1986) Robot accuracy analysis based on kinematics IEEE Journal on Robotics and Automation 2: 171-179.

4. Roth ZVIS, Mooring B, Ravani B (1987) An overview of robot calibration. IEEE Journal on Robotics and Automation 3: 377-385.

5. Schroer K, Albright SL, Grethlein M (1997) Complete, minimal and modelcontinuous kinematic models for robot calibration. Robotics and ComputerIntegrated Manufacturing 13: 73-85.

6. Kamali K, Joubair A, Bonev IA, Bigras P (2016) Elasto-geometrical calibration of an industrial robot under multidirectional external loads using a laser tracker. 2016 IEEE International Conference on Robotics and Automation (ICRA). Stockholm, Sweden.

7. Jin J, Gans N (2015) Parameter identification for industrial robots with a fast and robust trajectory design approach. Robotics and Computer-Integrated Manufacturing 31: 21-29

8. Joubair A, Bonev IA (2015) Kinematic calibration of a six-axis serial robot using distance and sphere constraints. The International Journal of Advanced Manufacturing Technology 77: 515-523

9. Hudgens J, Cox D, Tesar D (2000) Classification structure and compliance modeling for serial manipulators. Proceedings 2000 ICRA. Millennium Conference. San Francisco, California, USA.

10. Judd RP, Knasinski AB (1990) A technique to calibrate industrial robots with experimental verification. IEEE Transactions on robotics and automation 6 : 20-30.

11. Duelen G, Schroer K (1991) Robot calibration-method and results. Robotics and Computer-Integrated Manufacturing 8: 223-231.

12. Wang Z, Liu R, Chen X, Sparks T, Liou F (2016) Industrial Robot Trajectory Stiffness Mapping for Hybrid Manufacturing Process. International Journal of Robotics and Automation Technology 3: 28-39. 
Citation: Wang Z, Liu R, Sparks T, Chen X, Liou F (2018) Industrial Robot Trajectory Accuracy Evaluation Maps for Hybrid Manufacturing Process Based on Joint Angle Error Analysis. Adv Robot Autom 7: 183. doi: 10.4172/2168-9695.1000183

Page 12 of 12

13. Ruggiu M (2012) Cartesian stiffness matrix mapping of a translational parallel mechanism with elastic joints. International Journal of Advanced Robotic Systems.

14. Pinto C, Corral J, Altuzarra O, Hernandez A (2010) A methodology for static stiffness mapping in lower mobility parallel manipulators with decoupled motions. Robotica 28: 719-735.

15. Hayat AA, Chittawadigi RG, Udai AD, Saha SK (2013) Identification of DenavitHartenberg parameters of an industrial robot. Proceedings of Conference on Advances In Robotics. Pune, India. 